\title{
Accessing eigenstate spin-glass order from reduced density matrices
}

\author{
Younes Javanmard, ${ }^{1}$ Soumya Bera, ${ }^{2}$ and Markus Heyl ${ }^{1}$ \\ ${ }^{1}$ Max-Planck-Institut für Physik komplexer Systeme, Nöthnitzer Straße 38, 01187-Dresden, Germany \\ ${ }^{2}$ Department of Physics, Indian Institute of Technology Bombay, Mumbai 400076, India
}

(Dated: June 8, 2018)

\begin{abstract}
Many-body localized phases may not only be characterized by their ergodicity breaking, but can also host ordered phases such as the many-body localized spin-glass (MBL-SG). The MBL$\mathrm{SG}$ is challenging to access in a dynamical measurement and therefore experimentally since the conventionally used Edwards-Anderson order parameter is a two-point correlation function in time. In this work, we show that many-body localized spin-glass order can also be detected from twosite reduced density matrices, which we use to construct an eigenstate spin-glass order parameter. We find that this eigenstate spin-glass order parameter captures spin-glass phases in random Ising chains both in many-body eigenstates as well as in the nonequilibrium dynamics from a local in time measurement. We discuss how our results can be used to observe MBL-SG order within current experiments in Rydberg atoms and trapped ion systems.
\end{abstract}

Introduction. - Recently, it has been proposed that phases of quantum many-body systems may not only be characterized in terms of their thermodynamic properties but also on the level of single eigenstates [1-8]. These so-called eigenstate phases are protected by nonergodicity where the long-time dynamical properties of the system cannot be captured by a thermodynamic ensemble. Consequently, systems can exhibit order in steady states resulting from real-time dynamics although the thermal states at the corresponding energy density are featureless. The protecting nonergodicity can be generated by strong quenched disorder [4, 7, 9-19] or dynamical constraints due to gauge invariance [20-22]. Recently, the dynamical signatures of such eigenstate phases have been probed in experiments including the observation of manybody localization (MBL) [23-28] or discrete time crystals $[29,30]$. However, some of the proposed eigenstate phases, such as the MBL spin glass (MBL-SG) $[1,2,8]$, remain challenging to access dynamically and thus also experimentally.

In this work we show that MBL-SG order can be detected from two-site reduced density matrices, which we use to construct an eigenstate spin-glass (ESG) order parameter. We find that this ESG order parameter captures MBL-SG phases both in eigenstates as well as in the nonequilibrium dynamics from a local in time measurement, which makes MBL-SG order accessible within current experiments in quantum simulators. In previous works MBL spin glass order in an eigenstate $|\Psi\rangle$ has been detected for spin-1/2 systems using an EdwardsAnderson (EA) order parameter $[1,31]$

$$
X_{\mathrm{EA}}=\frac{1}{L^{2}} \sum_{i, j=1}^{L}\left\langle\Psi\left|\sigma_{i}^{z} \sigma_{j}^{z}\right| \Psi\right\rangle^{2},
$$

where $\sigma_{i}^{z}, i=1, \ldots, L$, denotes Pauli matrices on site $i$ with $L$ the total number of lattice sites. This order parameter requires access to single quantum many-body eigenstates, which experimentally is not achievable and also limits numerical studies to exact diagonalization and therefore the reachable system sizes. While $X_{\mathrm{EA}}$ can also be rewritten in the time domain, a measurement of $X_{\mathrm{EA}}$ then requires access to a two-time correlation function at large times, which is experimentally challenging and not possible within current quantum simulator implementations.

Eigenstate spin-glass order parameter. - For the definition of the eigenstate spin-glass order parameter $X_{\mathrm{ESG}}$, let us first fix two lattice sites $i$ and $j$. Moreover, let us denote the reduced density matrix of these two sites by $\varrho_{i j}$, which can be obtained from the full density matrix $\varrho$ by tracing out the complement of the two sites $i$ and $j$. The main idea behind $X_{\mathrm{ESG}}$ is to not calculate the square of the spin-spin correlator in Eq. (1) for the full quantum many-body eigenstate, but rather on the local equivalent which are the eigenstates of the reduced density matrix $\varrho_{i j}$. Accordingly, we diagonalize the $4 \times 4$ ma$\operatorname{trix} \varrho_{i j}=\sum_{n} p_{n j}^{i f}\left|\psi_{n}^{i j}\right\rangle\left\langle\psi_{n}^{i j}\right|$ to find its eigenvalues $\left(p_{n}^{i j}\right)$, eigenvectors $\left(\left|\psi_{n}^{i j}\right\rangle\right)$ and calculate the following quantity:

$$
\chi_{\mathrm{ESG}}^{i j}=\sum_{n=1}^{4} p_{n}^{i j}\left\langle\psi_{n}^{i j}\left|\sigma_{i}^{z} \sigma_{j}^{z}\right| \psi_{n}^{i j}\right\rangle^{2} .
$$

Finally, we perform a spatial average over all pairs $(i, j)$ via:

$$
x_{\mathrm{ESG}}=\frac{1}{L(L-1)} \sum_{i \neq j}^{L} X_{\mathrm{ESG}}^{i j} .
$$

It is the central result of this work that for a paradigmatic MBL spin-glass model the $\mathcal{X}_{\mathrm{ESG}}$ detects the eigenstate spin-glass order as we show in detail below. Thus, MBL spin-glass order doesn't require knowledge of the full quantum many-body eigenstate, but rather only the local information contained in the reduced density matrix. We compare $X_{\mathrm{ESG}}$ and $X_{\mathrm{EA}}$ both in the GS for large systems using DMRG and in highly-excited states using exact diagonalization. We find numerical evidence that both of these quantities are not only quantitatively close but also can be used as order parameters for the MBL 
spin glass transition in the studied model. Importantly, we also show that $X_{\text {ESG }}$ can be used as a dynamical measure to detect the MBL spin-glass order. In particular, we find that for typical initial conditions, the long-time limit of $X_{\mathrm{ESG}}$ is nonzero in the ordered phase and vanishes in the paramagnetic one. However, towards the transition the dynamics becomes very slow such that accessing the structure of the transition remains challenging. In the end we will discuss how to observe our findings in current experiments.

Model and method. - We study the ESG order parameter $x_{\text {ESG }}$ for the following quantum Ising chain with open boundary conditions,

$$
\hat{\mathcal{H}}=-\frac{1}{2}\left[\sum_{i=1}^{L-1} J_{i}^{z} \sigma_{i}^{z} \sigma_{i+1}^{z}+\sum_{i=1}^{L-1} J_{i}^{x} \sigma_{i}^{x} \sigma_{i+1}^{x}+\sum_{i=1}^{L} h_{i}^{x} \sigma_{i}^{x}\right],
$$

where $\sigma_{i}^{x, z}, i=1, \ldots, L$ are the Pauli matrices and $L$ denotes the total number of lattice sites. All the parameters appearing in this model are random and taken from uniform distributions. We choose $J_{i}^{z} \in[-J, J]$ and $h_{i}^{x} \in[-h, h]$ from uniform box distribution. For vanishing $J_{i}^{x}$ the model reduces to the transverse field Ising chain, which is integrable and exactly solvable by a mapping to a quadratic fermionic theory using a JordanWigner transformation [32]. To make the model generic and non-integrable we add a weak random $J_{i}^{x} \in\left[-\frac{h}{4}, \frac{h}{4}\right]$ term, which becomes equivalent to a two-particle interaction in the fermionic language and renders the model non-integrable.

The transverse-field Ising chain with $J_{i}^{x}=0$ exhibits a $T=0$ quantum phase transition from a paramagnetic $(J<h)$ state to a doubly degenerate spin-glass ground state $(J>h)$ [33]. In order to explore the ground state physics for the interacting model at $J_{i}^{x} \neq 0$, we use density-matrix renormalization group (DMRG) techniques within a matrix product state formulation [34] and used second order Suzuki-Trotter decomposition to exponentiate the unitary operator [35]. This allows us to probe the phase transition for large system sizes reducing finite-size effects. In order to access the high energy eigenstates we use standard exact diagonalization and typically calculate 16 eigenstates from the middle of the spectrum and perform an average of $X_{\mathrm{ESG}}$ over this set of states. At excited energies around $\sim 1000$ disorder configurations are used to perform statistical averaging of $X_{\mathrm{EA}}$ and $X_{\mathrm{ESG}}$, while in ground state $\sim 100$ disorder realizations are considered for averaging. Finally, for simulating the dynamics for large system sizes the timeevolving block decimation technique is used. To minimize finite-size effects, we calculate $X_{\mathrm{EA}}$ and $x_{\mathrm{ESG}}$ by averaging not over all pairs $(i, j)$ of lattice sites but rather restrict to those pairs with $|i-j|>4$. Also to minimize the edge effects for such small system sizes we excluded the edge site contribution in $X_{\mathrm{EA}}$ and $X_{\mathrm{ESG}}$.

Eigenstate results. - First we study the $X_{\mathrm{ESG}}$ in eigenstates and compare it to the Edwards-Anderson order pa-
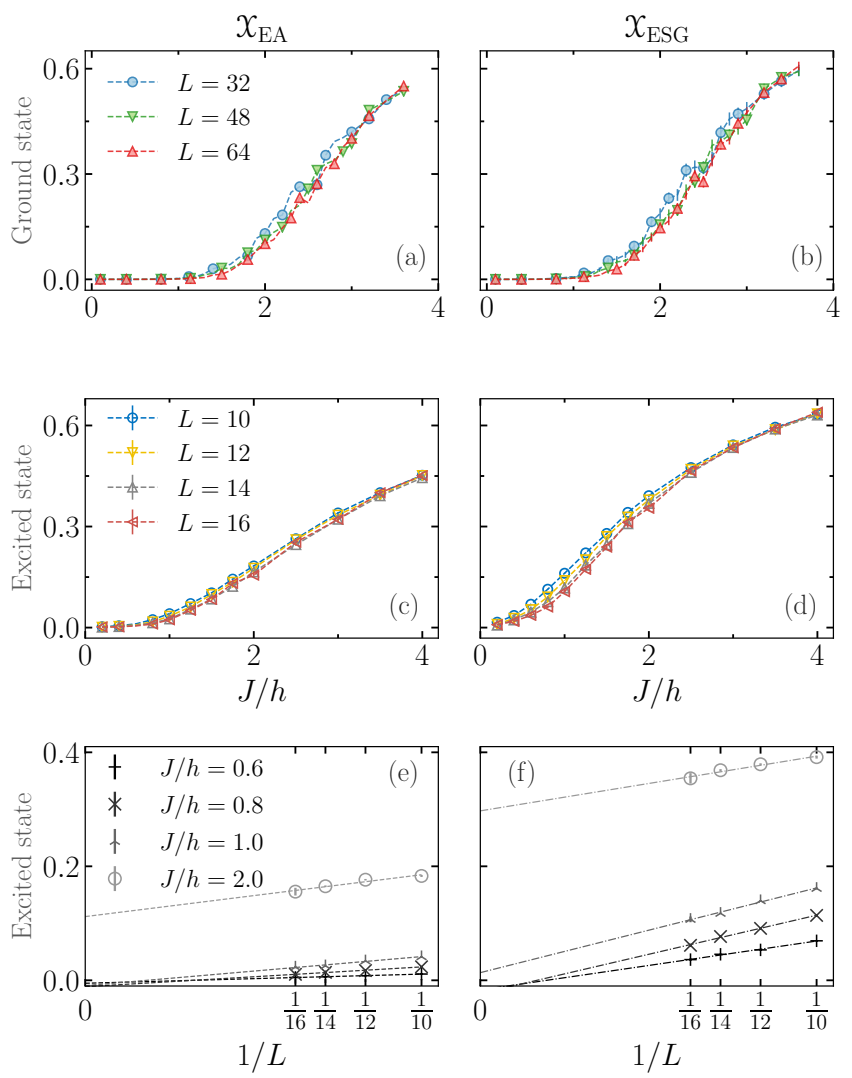

FIG. 1. Eigenstate spin-glass order. Upper panel: (a) Shows the Edwards-Anderson order parameter, $X_{\mathrm{EA}}$, in the ground state of the Hamiltonian Eq. (4) obtained using DMRG for system sizes $L=32,40,48$ as a function of coupling strength $J / h$. While for weak couplings $X_{\mathrm{EA}}$ approaches zero, for large $J / h \gtrsim 1.5 X_{\mathrm{EA}}$ becomes nonvanishing in the expected spin-glass ordered phase. (b) The eigenstate spinglass order parameter $X_{\mathrm{ESG}}$ in the ground state for the same system sizes. (c) $X_{\mathrm{EA}}$ in excited states from the center of the spectrum for system sizes $L=10,12,14,16$ obtained from exact diagonalization, and compared to $X_{\mathrm{ESG}}(\mathrm{d})$. In (e-f) we show the finite-size dependence of $\mathcal{X}_{\mathrm{ESG}}$ for excited states. For couplings $J / h \lesssim 1, X_{\mathrm{ESG}}$ tends towards a vanishing value for increasing system sizes, whereas for $J / h \gtrsim 1$ it is large and nonzero. The included lines are a guide to the eye and don't represent a quantitative extrapolation.

rameter $x_{\mathrm{EA}}$. In Fig. $1(\mathrm{a}-\mathrm{d})$ we plot both $x_{\mathrm{EA}}$ and $x_{\mathrm{ESG}}$ for the ground state calculated using the density-matrix renormalization group for system sizes $L=32,48,64$ and exact diagonalization for $L=8,12,16$ as a function of the spin-spin coupling strength strength $J / h$. The top panel shows the ground state results. As expected, for weak couplings $(J / h<1.5)$ the system is in a paramagnetic phase and thus $X_{\mathrm{EA}}$ vanishes. The $X_{\mathrm{ESG}}$ is showing an analogous behavior, as can be seen in Fig. 1(b). In the spin-glass phase $x_{\mathrm{EA}}$ is finite and almost independent of systems size, as we find also for $X_{\mathrm{ESG}}$, see Fig. 1(b). Overall, these results suggest that the $\mathcal{X}_{\mathrm{ESG}}$ parameter can be taken as an order parameter for the spin-glass quantum phase transition in the considered model. 
In Fig. 1(c-d) we study spin-glass order in excited states of the same model Eq. (4) where we observe an overall similar behavior. For sufficiently weak couplings both $x_{\mathrm{EA}}$ and $x_{\mathrm{ESG}}$ take small values indicating that the system does not exhibit MBL-SG order. This is different for large couplings where both $X_{\mathrm{EA}}$ and $X_{\mathrm{ESG}}$ saturate to a large nonzero value almost independent of system size. More quantitatively, we analyze the finite size dependence for strong and weak couplings by plotting both the eigenstate order parameters as a function of $1 / L$ for several disorder values $(J / h=0.6,0.8,1.0,2.5)$ in Fig. $1(\mathrm{e}-\mathrm{f})$. While for large couplings both $X_{\mathrm{EA}}$ and $X_{\mathrm{ESG}}$ are almost independent of system size, at weak couplings a linear extrapolation in $1 / L$ suggests vanishing values. This extrapolation should be taken only as a guide to the eye as the precise functional form of the $L$-dependence is not known. Due to the limited system sizes and the resulting strong finite-size effects we don't attempt to extract the MBL-SG transition from the exact diagonalization data. We find, however, that the measurement of $X_{\mathrm{ESG}}$ in the real-time dynamics, as discussed in the following, is much better suited for that purpose.

Quench dynamics. - It is a crucial observation that $\chi_{\mathrm{ESG}}$ can be computed for any state. In particular, we now show that this makes it possible to monitor the buildup of MBL-SG order in the quantum real-time dynamics from a local in time measurement. This observation is not only useful from a theoretical point of view, but also makes the observation of MBL-SG phases accessible for current experiments in quantum simulators.

In the following we study the real-time evolution of the ESG order parameter $X_{\mathrm{ESG}}(t)$ from initial spin configurations with random orientations along the $\sigma^{x}$ direction therefore respecting the $\mathbb{Z}_{2}$ symmetry of the Hamiltonian. In the supplementary material we also show data for random initial spin configurations aligned along the $\sigma^{z}$ direction, which break the $\mathbb{Z}_{2}$ symmetry [36]. We compute the dynamics using time dependent DMRG (tDMRG) using the second order Trotter decomposition with $d t=0.01$, where we kept only those states with singular values above $10^{-9}$. An analysis of the numerical accuracy and time steps in tDMRG calculations can be found in the supplementary material [36].

In Fig. 2 we show the dynamical evolution of $\chi_{\mathrm{ESG}}$, where we compare two representatives for the temporal behavior for weak couplings in Fig. 2a and for strong couplings in Fig. 2b, respectively. At $t=0$ we have that $X_{\mathrm{ESG}}(t=0)=0$ since the initial condition is structureless and does not contain any spatial correlations. In the transient stage of the dynamics we observe an increase of $x_{\mathrm{ESG}}(t)$ to nonzero values as a consequence of an initial buildup of spatial correlations. On longer time scales two qualitatively different dynamical regimes emerge depending on the coupling strength, suggesting that MBLSG order can be detected from the long-time limit of $x_{\mathrm{ESG}}(t)$. While for weak couplings $X_{\mathrm{ESG}}(t)$ decays for increasing time, this is not the case for strong couplings, where $X_{\mathrm{ESG}}(t)$ saturates to a nonzero value.
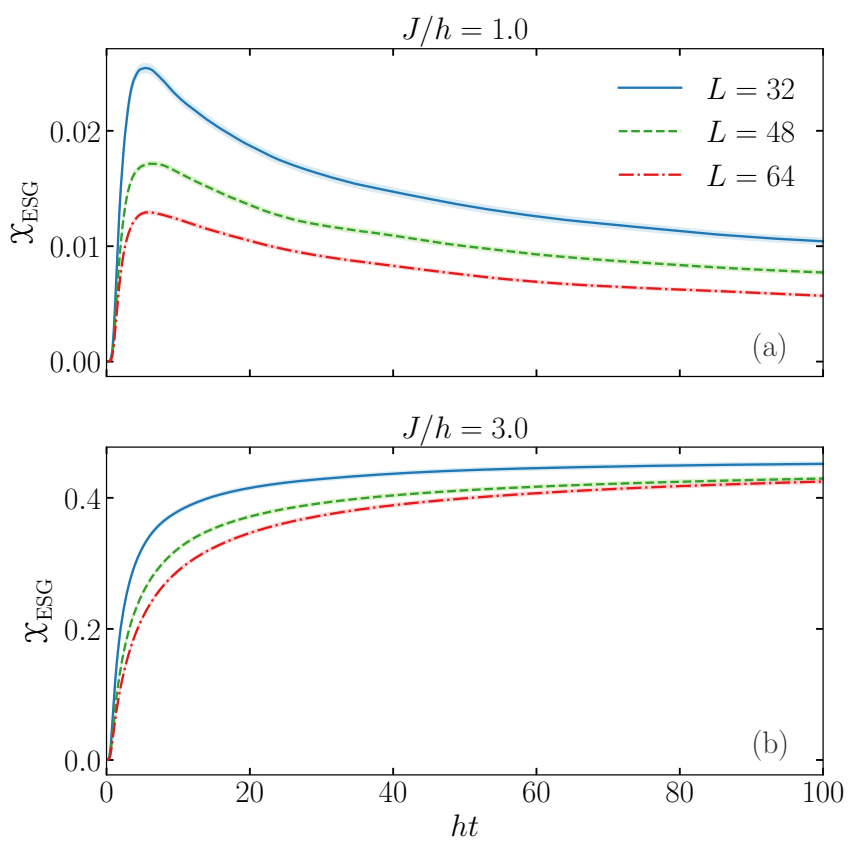

FIG. 2. Quench dynamics. Dynamical evolution of $X_{\mathrm{ESG}}$ starting from a state where all spins are initially aligned along the transverse direction. (a) For weak couplings $J / h=1.0$ $X_{\mathrm{ESG}}$ is small and decays on long-time scales. (b) For larger couplings in the eigenstate spin-glass ordered phase $J / h=$ 3.0, $x_{\mathrm{ESG}}$ increases steadily with time approaching a nonzero value in the long-time limit. The shaded region indicates the statistical error in the data due to a finite set of disorder averages.

Figure 3 demonstrates how one could possibly identify the MBL-SG transition dynamically. In each of the panels of this figure we plot for different system size the value of $X_{\mathrm{ESG}}$ as a function of the coupling strength and how this value changes as a function of time $t$. For each $L$ one can identify two dynamical regimes, one where $X_{\mathrm{ESG}}$ increases and one where $\mathcal{X}_{\mathrm{ESG}}$ decays as a function of time within accessible time scales in numerical simulation. We identify the regime where $X_{\mathrm{ESG}}$ goes to a nonzero value as the MBL-SG phase. From the plots in Fig. 3 we observe a crossing point for each system size which suggest that it might be possible to estimate the critical coupling strength for the MBL-SG transition using $X_{\mathrm{ESG}}$. From our current data, however, this does not appear to be possible accurately. For the exactly solvable case $J_{i}^{x}=0$, it is well known that the MBL spin-glass transition is located at $J / h=1$ [32]. Computing with tDMRG the dynamics for the same system sizes and times, we find that in this case the crossing point is located around $J \approx 1.2$ [36], which overestimates the region of the MBL paramagnet. We attribute this observation to the slow expected dynamics in these models, which can show slow power-law or also logarithmic relaxation [37]. While the crossing point for the times accessible within tDMRG shows only a weak dependence on time, it is very likely that it exhibits a further slow drift on even longer times 

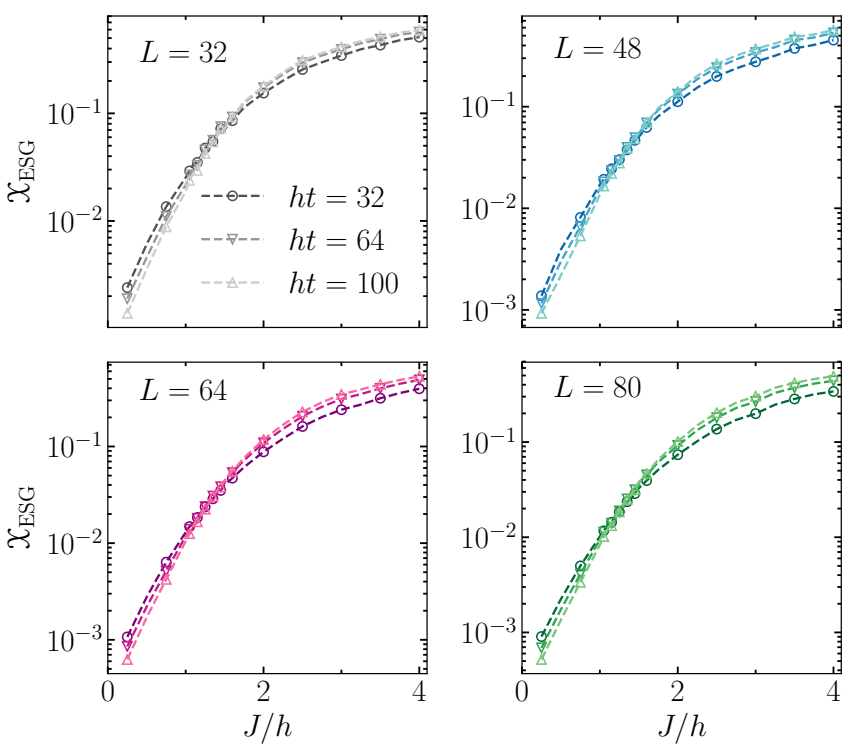

FIG. 3. Eigenstate spin-glass order parameter across the transition. Time evolution of $\mathcal{X}_{\mathrm{ESG}}$ as a function of disorder strength $J / h$ for different system sizes, $L=32,48,64,80$ in different panels. For weak couplings $X_{\text {ESG }}$ decays with time, while in the opposite regime it increases. A crossing point separates these regimes of opposite dynamical behavior.

scales.

Discussion. - In this work we have provided evidence that the detection of MBL spin-glass order does not require access on the full many-body eigenstates, as is necessary for the previously used Edwards-Anderson order parameter. We rather find that MBL spin-glass order in random quantum Ising chains is contained in two-spin reduced density matrices.

This observation has several implications. On the theory side, reduced density matrices can be accessed with a variety of methods, whereas full eigenstates require in general the use of exact diagonalization, which a priori limits the accessible system sizes. We have shown in this work, for example, that our proposed eigenstate spinglass (ESG) order parameter can be computed using the density-matrix renormalization group method, which allows us to reach systems up to at least 80 spins. This can be achieved, since the ESG can be obtained from a local in time measurement in contrast to the EdwardsAnderson order parameter, which either requires access to the full eigenstates or to a two-time correlation function.

The property, that the ESG can be obtained by a local in time measurement, not only makes MBL spin-glass order theoretically more easily accessible, but also makes an experimental detection more feasible. Still, the ESG requires the full reconstruction of a reduced density matrix of two spins. While this limits the range of applicable experimental platforms, reduced density matrices are accessible in so-called quantum simulators such as trapped ions [38, 39], superconducting qubits [40], Rydberg systems, or ultra-cold atoms in optical lattices [41]. The observation of MBL spin-glass order remains nevertheless challenging since the experimental realization of system Hamiltonians, that are capable to host MBL spin-glass ordered phases, has not yet been reported. For trapped ions systems, however, a way to generate an Ising Hamiltonian with random spin interactions has been proposed $[42,43]$. In general, random interactions might also be straightforwardly realized using the digital approach to quantum simulation $[40,44,45]$ which is currently limited, however, by the accessible system sizes.

We have analyzed the ESG for a random quantum Ising chain, so that it is a natural question to which extent our results generalize to other models. While addressing this question on general grounds is beyond of the scope of this work, in the supplementary material we also show numerical results for a long-range Ising model with algebraically decaying spin-spin interactions, whose realization in a trapped ion system appears feasible within current experimental techniques. While finite-size effects in this model are stronger than for the nearest-neighbor Ising chain, we find a similar behavior of the EdwardsAnderson order parameter and the ESG, indicating that our results extend beyond the particular quantum Ising chain studied here.

Acknowledgments. - We would like to thank J. H. Bardarson and Talía L. M. Lezama for several discussions. SB acknowledges support from DST, India, through Ramanujan Fellowship Grant No. SB/S2/RJN-128/2016, and $\mathrm{MH}$ by the Deutsche Forschungsgemeinschaft via the Gottfried Wilhelm Leibniz Prize program. SB also acknowledges hospitality of the Max-Planck Institute for the Physics of Complex Systems, Dresden, Germany during the completion of the work.
[1] D. A. Huse, R. Nandkishore, V. Oganesyan, A. Pal, and S. L. Sondhi, Phys. Rev. B 88, 014206 (2013).

[2] J. A. Kjäll, J. H. Bardarson, and F. Pollmann, Phys. Rev. Lett. 113, 107204 (2014).

[3] A. Chandran, V. Khemani, C. R. Laumann, and S. L. Sondhi, Phys. Rev. B 89, 144201 (2014).

[4] B. Bauer and C. Nayak, J. Stat. Mech. Theor. Exp. (2013).
[5] D. Pekker, G. Refael, E. Altman, E. Demler, and V. Oganesyan, Phys. Rev. X 4, 011052 (2014).

[6] Y. Bahri, R. Vosk, E. Altman, and A. Vishwanath, Nat. Comm. 6, 7341 (2015).

[7] R. Nandkishore and D. A. Huse, Annu. Rev. Condens. Matter Phys. 6, 15 (2015).

[8] S. A. Parameswaran, A. C. Potter, and R. Vasseur, Ann. Phys. (Berl.) 529, 1600302 (2017). 
[9] I. V. Gornyi, A. D. Mirlin, and D. G. Polyakov, Phys. Rev. Lett. 95, 206603 (2005).

[10] D. Basko, I. Aleiner, and B. Altshuler, Ann. Phys. 321, 1126 (2006).

[11] M. Žnidarič, T. c. v. Prosen, and P. Prelovšek, Phys. Rev. B 77, 064426 (2008).

[12] A. Pal and D. A. Huse, Phys. Rev. B 82, 174411 (2010).

[13] J. H. Bardarson, F. Pollmann, and J. E. Moore, Phys. Rev. Lett. 109, 017202 (2012).

[14] E. Altman and R. Vosk, Annu. Rev. Condens. Matter Phys. 6, 383 (2014).

[15] D. J. Luitz, N. Laflorencie, and F. Alet, Phys. Rev. B 91, 081103 (2015).

[16] S. Bera, H. Schomerus, F. Heidrich-Meisner, and J. H. Bardarson, Phys. Rev. Lett. 115, 046603 (2015).

[17] Y. Bar Lev, G. Cohen, and D. R. Reichman, Phys. Rev. Lett. 114, 100601 (2015).

[18] S. Bera, G. De Tomasi, F. Weiner, and F. Evers, Phys. Rev. Lett. 118, 196801 (2017).

[19] F. Alet and N. Laflorencie, (2017), arXiv:1711.03145.

[20] A. Smith, J. Knolle, D. L. Kovrizhin, and R. Moessner, Phys. Rev. Lett. 118, 266601 (2017).

[21] A. Smith, J. Knolle, R. Moessner, and D. L. Kovrizhin, Phys. Rev. Lett. 119, 176601 (2017).

[22] M. Brenes, M. Dalmonte, M. Heyl, and A. Scardicchio, Phys. Rev. Lett. 120, 030601 (2018).

[23] M. Schreiber, S. S. Hodgman, P. Bordia, H. P. Lüschen, M. H. Fischer, R. Vosk, E. Altman, U. Schneider, and I. Bloch, Science 349, 842 (2015).

[24] M. Ovadia, D. Kalok, I. Tamir, S. Mitra, B. Sacépé, and D. Shahar, Sci. Rep. 5, 13503 EP (2015).

[25] J. Smith, A. Lee, P. Richerme, B. Neyenhuis, P. W. Hess, P. Hauke, M. Heyl, D. A. Huse, and C. Monroe, Nat. Phys. advance online publication (2016).

[26] J. yoon Choi, S. Hild, J. Zeiher, P. Schauß, A. RubioAbadal, T. Yefsah, V. Khemani, D. A. Huse, I. Bloch, and C. Gross, Science 352, 1547 (2016).

[27] P. Bordia, H. P. Lüschen, S. S. Hodgman, M. Schreiber, I. Bloch, and U. Schneider, Phys. Rev. Lett. 116, 140401 (2016).

[28] A. Lukin, M. Rispoli, R. Schittko, M. E. Tai, A. M. Kaufman, S. Choi, V. Khemani, J. Léonard, and M. Greiner, ArXiv e-prints (2018), arXiv:1805.09819 [cond-mat.quant-gas].

[29] Zhang J., Hess P. W., Kyprianidis A., Becker P., Lee A., Smith J., Pagano G., Potirniche I.-D., Potter A. C., Vishwanath A., Yao N. Y., and Monroe C., Nature 543, 217 (2017).

[30] Choi Soonwon, Choi Joonhee, Landig Renate, Kucsko Georg, Zhou Hengyun, Isoya Junichi, Jelezko Fedor, Onoda Shinobu, Sumiya Hitoshi, Khemani Vedika, von Keyserlingk Curt, Yao Norman Y., Demler Eugene, and Lukin Mikhail D., Nature 543, 221 (2017).

[31] K. Binder and A. P. Young, Rev. Mod. Phys. 58, 801 (1986).

[32] P. Pfeuty, Ann. Phys. 57, 79 (1970).

[33] S. Sachdev, Quantum Phase Transitions, 2nd ed. (Cambridge University Press, 2011).

[34] U. Schollwck, Ann. Phys. 326, 96 (2011).

[35] M. Suzuki, Progr. Theor. Phys. 56, 1454 (1976).

[36] See supplemental material for further numerical details, and convergence tests of the eigenstate order.

[37] R. Vosk and E. Altman, Phys. Rev. Lett. 110, 067204 (2013).
[38] R. Blatt and C. F. Roos, Nat. Phys. 8, 277 (2012), 0905.0118.

[39] P. Jurcevic, B. P. Lanyon, P. Hauke, C. Hempel, P. Zoller, R. Blatt, and C. F. Roos, Nature 511, 202 (2014).

[40] R. Barends, A. Shabani, L. Lamata, J. Kelly, A. Mezzacapo, U. L. Heras, R. Babbush, A. G. Fowler, B. Campbell, Y. Chen, Z. Chen, B. Chiaro, A. Dunsworth, E. Jeffrey, E. Lucero, A. Megrant, J. Y. Mutus, M. Neeley, C. Neill, P. J. J. O'Malley, C. Quintana, P. Roushan, D. Sank, A. Vainsencher, J. Wenner, T. C. White, E. Solano, H. Neven, and J. M. Martinis, Nature 534, 222 (2016).

[41] L. A. Pena Ardila, M. Heyl, and A. Eckardt, In preparation.

[42] P. Hauke, L. Bonnes, M. Heyl, and W. Lechner, Frontiers in Physics 3, 21 (2015).

[43] T. Graß, D. Raventós, B. Juliá-Díaz, C. Gogolin, and M. Lewenstein, Nat. Commun. 7, 11524 (2016).

[44] B. P. Lanyon, C. Hempel, D. Nigg, M. Müller, R. Gerritsma, F. Zaehringer, P. Schindler, J. T. Barreiro, M. Rambach, G. Kirchmair, M. Hennrich, P. Zoller, R. Blatt, and C. F. Roos, Science 334, 57 (2011).

[45] E. A. Martinez, C. A. Muschik, P. Schindler, D. Nigg, A. Erhard, M. Heyl, P. Hauke, M. Dalmonte, T. Monz, P. Zoller, and R. Blatt, Nature 534, 516 (2016).

[46] D. Gobert, C. Kollath, U. Schollwöck, and G. Schütz, Phys. Rev. E 71, 036102 (2005). 


\section{SUPPLEMENTARY INFORMATION: ACCESSING EIGENSTATE SPIN-GLASS ORDER FROM REDUCED DENSITY MATRICES}

In this supplementary document we provide additional data and describe the required error analysis for the real time evolution using the time evolving block decimation algorithm.

\section{REAL TIME EVOLUTION USING TIME EVOLVING BLOCK DECIMATION (TEBD) TECHNIQUE}

to We use the standard TEBD technique [34] to perform unitary real time evolution starting from a given initial state.

$$
|\psi(t)\rangle=U(t)\left|\psi_{\text {in }}(0)\right\rangle=e^{-i H t}\left|\psi_{\text {in }}(0)\right\rangle,
$$

where $H$ is the Hamiltonian of the system considered. The TEBD algorithm relies on the Suzuki-Trotter decomposition of the time-evolution operator $U(t)$, Eq. S1. In this context one decomposes the time-evolution operator into $N$ small time steps, where $N$ is a large enough number such that the time interval $d t=\frac{t}{N}$ is small compared to the physical time scale of the system.

$$
U(t)=U(d t=t / N)^{N} .
$$

Due to the local nature of the Hamiltonian, i.e., only nearest-neighbor interaction, the Hamiltonian can be conveniently decomposed into sum of many local terms $h_{i}$ with support only on lattice sites $i$ and $i+1$. Therefore $U(d t)$ can be approximated by an $n$ th-order Trotter decomposition [35]. In our calculations we use a second order Suzuki-Trotter decomposition, where the local terms can be broken down to product of terms in even and odd sutes,

$$
U(d t)=\prod_{\substack{i \\
\text { even }}} U_{i}\left(\frac{d t}{2}\right) \prod_{\substack{i \\
\text { odd }}} U_{i}(d t) \prod_{\begin{array}{c}
i \\
\text { even }
\end{array}} U_{i}\left(\frac{d t}{2}\right)+O\left(d t^{3}\right) .
$$

Where the $U_{n}(d t)$ are the infinitesimal time-evolution operators $\exp \left(-i h_{i, i+1} d t\right)$ on the bonds $i$ which can be even or odd.

\section{A. Error sources and analysis}

There are two main sources of error that one needs to take into account while analysing the time-dependent data.

a. Trotter error: Due to finite degree of Trotter decomposition an error accumulates in time evolution. For an $n$ th-order Trotter decomposition, the error in one time step $d t$ is of order $d t^{n+1}$. To reach a given time $t$, one has to perform $t / d t$ time steps, such that in the worst case the error grows linearly in time $t$ and the resulting error is of order $(d t)^{n} t$. As well in our computation the error scales linearly with system size $L$, which is due to $2 \times L / 2$ number of Trotter gate operation for given $d t$. Therefore the overall error is of the order $(d t)^{n} L t$.

b. Truncation error: This error arises due to truncation of local matrices due to ever growing Hilbert space during the TEBD run. The truncation error $\epsilon$ at each time step is small but it accumulates as $O(L t / d t)$ for total time $t$. This is due to the fact that truncated wave function after each time step $d t$ has a norm less than 1 and needs to be renormalized by a factor of $(1-\epsilon)^{-1}>1$. Truncation errors accumulate roughly exponentially with an exponent of $\epsilon L t / d t$ and eventually the calculations break down at very large time limit due to error propagation. Therefore carefully checking the error at each step and accommodating sufficient number of states to control it is necessary.

c. Optimal choice of TEBD parameters: In order to reach long simulation time $t$ one has to find optimal control parameters, which are time step $d t$, and the number of the truncated states (kept state) $\chi_{\max }$. We implemented the TEBD algorithm is such a way that we discarded states below certain threshold, $\varepsilon$.

Therefore the control parameters are the time step $d t$ and the truncation error threshold is $\varepsilon$. The total error would increase at larger $d t$ due to the Trotter error, and at smaller $d t$ due to the truncation error. It is reasonable to choose for small times rather small values of $d t$ in order to minimize the Trotter error and for large times, to choose a somewhat coarser time interval, in order to push the time to as large as possible [46]. We choose two small value for $d t$

$$
h d t \in[0.01,0.005]
$$

and for $\varepsilon$ we consider different truncation thresholds

$$
\varepsilon \in[1 e-7,1 e-9,1 e-11]
$$



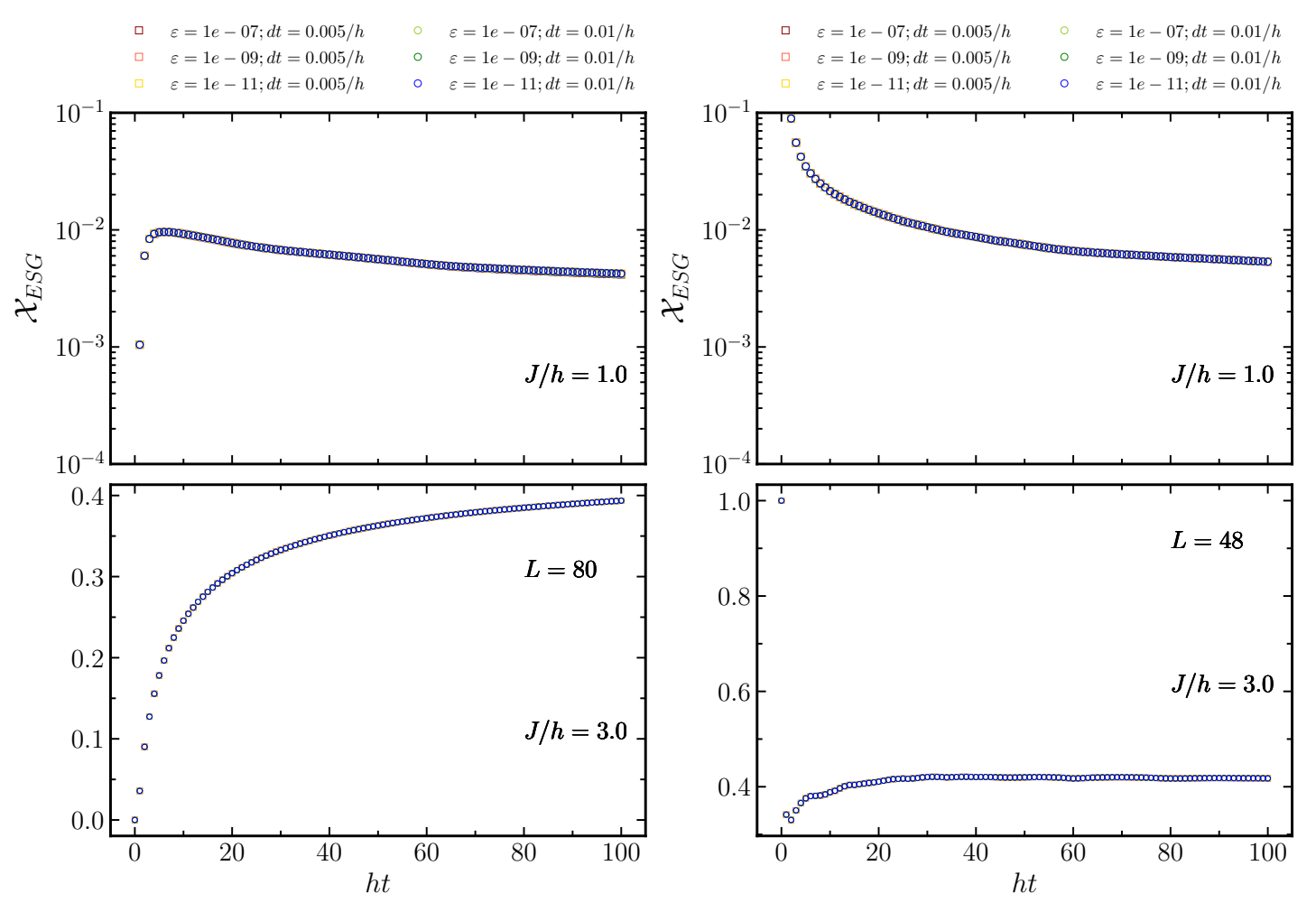

FIG. S1. Error analysis for different set of parameters $(\varepsilon, d t)$ for non-MBL SG, $J / h=1.0$, and MBL-SG, $J / h=3.0$, phases. Left pannel for a chain $L=80$ with all initial states randomly pointed in the $x$-direction. Right pannel for a chain $L=48$ with all initial states randomly pointed in the $z$-direction.

In the Following we do the error analysis for two different initial states, first error analysis for the results that we have shown in the main text of the paper and second for initial state that break the $Z_{2}$ symmetry of the system in the $z$-direction, i.e., an initial state where all spins are pointed randomly in the $z$-direction.

Figure S1 left panel shows the error analysis for the initial states, which is product state randomly directed in $x$ direction. Note that we average over 1000 realizations for this simulation. In each set of TEBD parameters, i.e., $\varepsilon$ and $d t$, the initial states remain unchanged, which give us confident that results shown here and in the main text are well converged.

Figure S1 right panel shows the evolution of the $\mathcal{X}_{\mathrm{ESG}}$ for different TEBD parameters starting with an initial state, which is randomly pointed in $z$-direction. As it is seen that for all the parameters choice of the TEBD algorithm the dynamics remain unaffected. Here the discarded weight remains very small of the order $1 e-16$.

\section{B. Integrable model: dynamics in the vicinity of the transition}

In Fig. S2 we show data for $X_{\mathrm{ESG}}$ in the vicinity of the transition for the integrable case of the model studied in the main text $\left(J^{x}=0\right)$, where the transition is known to be at $J / h=1$ [32]. From this plot one can again identify two phases separated by a crossing point, with which one might identify the location of the MBL-SG transition in the asymptotic long-time limit. From our finite-time data, however, we find the crossing point at $J / h \approx 1.2$, which overestimates the transition by $20 \%$. As in the main text, we attribute this discrepancy to the slow dynamics that can occur in these models, which effectively implies that one would need to reach even longer times to see a drift towards the known transition value.

\section{INITIAL STATE WITH BROKEN $Z_{2}$ SYMMETRY}

In this section we show additional data for quench dynamics when the initial state breaks the Ising $\mathbb{Z}_{2}$ symmetry by choosing product states aligned along the $z$-direction. As seen in Fig. S3 we observe the same long-time dynamics 


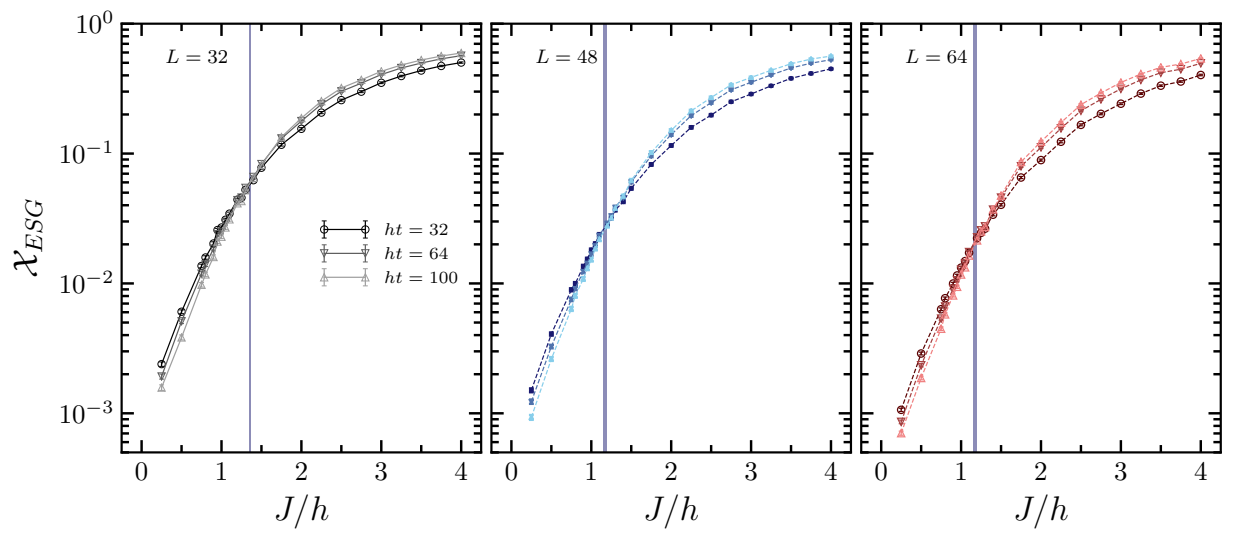

FIG. S2. Eigenstate spin-glass order parameter $X_{\mathrm{ESG}}$ across the transition in the integrable model, where the coupling along the $x$-direction is taken to be zero $\left(J^{x}=0\right.$, see main text for further details of the model). For weak couplings $X_{\mathrm{ESG}}$ decays with time, while in the other regime it increases.
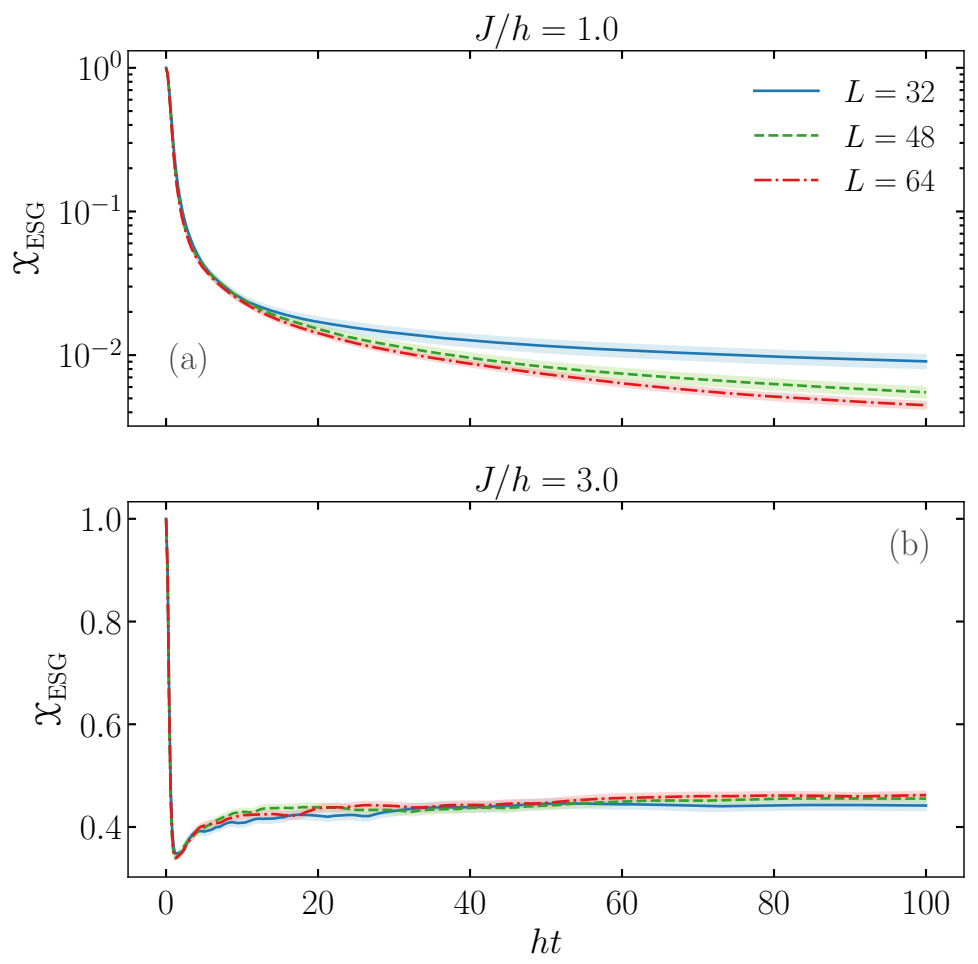

FIG. S3. Quench dynamics (a) Shows the dynamical evolution of $\mathcal{X}_{\text {ESG }}$ starting from an initial state where all spins initially align along the $z$-direction. Because of the initially broken $\mathbb{Z}_{2}$ symmetry at $t=0$, the $X_{\mathrm{ESG}}$ is non-zero as seen in both the plots. For weak coupling $J / h=1.0$ the $X_{\mathrm{ESG}}$ decays at long times. (b) For strong couplings $J=3.0$ the $X_{\mathrm{ESG}}$ becomes finite instead. The shaded region indicates the statistical error in the data.

as for the initial condition studied in the main text.For weak couplings the $X_{\mathrm{ESG}}$ values goes to zero with increasing system sizes at long times. For large couplings instead, $X_{E S G}$ remains finite at long times for all system sizes. 

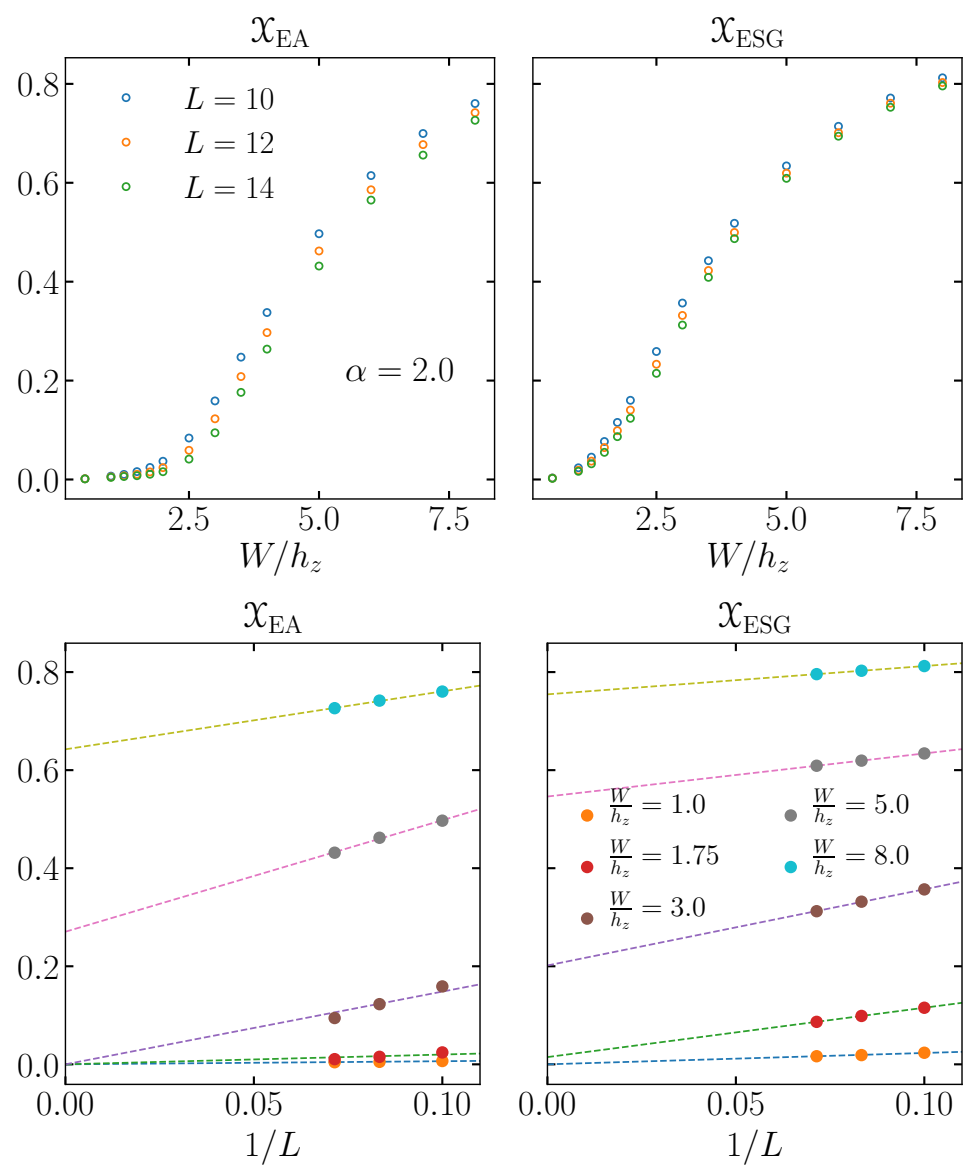

FIG. S4. Eigenstate order Upper panel: Shows both the $X_{\mathrm{EA}}$ and $\mathcal{X}_{\mathrm{ESG}}$ for the long-range model (S6) in for excited states taken from the middle of the spectrum. Above $W / h_{z} \gtrsim 2.5$ the model appears to have a nonzero $\mathcal{X}_{\mathrm{EA}}$, suggesting MBL-SG order. This behavior is also captured by the $X_{\mathrm{ESG}}$ as well. Lower panel shows the $L$ dependence of both the order parameters for few different values of disorder strength as mentioned in the label. Here $\alpha=2$ is chosen. As in the main text, the lines are a guide to the eye and are not supposed to provide a quantitative extrapolation.

\section{LONG-RANGE CORRELATED ISING MODEL}

In this section we consider a different model and show that also in the presence of long-range interaction the eigenstate order parameter shows the essential behavior of MBL-SG. The model we consider is the following:

$$
\mathcal{H}=\frac{1}{N(\alpha)} \sum_{i<j} \frac{\Omega_{i} \Omega_{j}}{|i-j|^{\alpha}} \sigma_{i}^{x} \sigma_{j}^{x}+\sum_{i} h_{i} \sigma_{i}^{z},
$$

where $N(\alpha)=\sum_{i<j} \frac{1}{|i-j|^{\alpha}}$ is the Kac normalization. $\Omega_{i}$ is taken from an uniform distribution [0,W], while the field is distributed randomly between $\left[-h_{z}, h_{z}\right]$. Like in the main text here we also calculated both the Edwards-Anderson parameter $X_{\mathrm{EA}}$ and the eigenstate order parameter $X_{\mathrm{ESG}}$. It has been theoretically proposed that this model can be realized in current trapped ion experiments [42, 43].

Figure S4 shows the parameter dependence of both $X_{\mathrm{EA}}$ and $X_{\mathrm{ESG}}$ along with the finite-size dependence. The calculation is performed for excited states taken from the middle of the spectrum for $\alpha=2.0$. As one can see the behavior of $X_{\mathrm{EA}}$ is well reproduced by the $X_{\mathrm{ESG}}$, however again due to strong finite size effects the critical disorder value for MBL-paramagnet to MBL-SG transition cannot be reliably detected. We have used similar averaging procedure as it is done for all the data in the main text. 